\title{
PNA on human sperm: a new approach for in situ aneuploidy estimation
}

\author{
Franck Pellestor ${ }^{*}, 1$ Brigitte Andréo ${ }^{1}$, Krihan Taneja $^{2}$ and Brett Williams ${ }^{2}$ \\ ${ }^{1}$ CNRS-UPR 1142, F-34396 Montpellier, France; ${ }^{2}$ Applied Biosystems, Bedford, MA, USA
}

Peptide nucleic acids (PNAs) are a relatively new class of synthetic DNA mimics based on a peptide-like backbone. Since their introduction, PNA probes have become established as an efficient variation on the standard FISH procedure for chromosomal identification. In this report we have experimented with centromeric PNA probes on human sperm preparations. Both $\mathrm{NaOH}$ and DTT sperm decondensation procedures have been tested and comparative estimates of disomies $\mathrm{X}, \mathrm{Y}$ and 1 have been performed in sperm from two donors using PNA, FISH and PRINS techniques. Similar results were obtained with the three methods, demonstrating the efficiency of PNA probes in the analysis of human sperm. The fast kinetics, stability and high specificity of PNA probes make PNA-based methodologies very valuable for in situ cytogenetic investigations.

European Journal of Human Genetics (2003) 11, 337-341. doi:10.1038/sj.ejhg.5200958

Keywords: PNA; human spermatozoa; aneuploidy; FISH; PRINS

\section{Introduction}

Direct chromosomal analysis of spermatozoa is an essential approach for the investigation of the occurrence and etiology of chromosomal abnormalities in humans under a wide variety of clinical conditions. To date, numerous chromosomal analyses on human sperm have been performed using fluorescence in situ hybridization (FISH) (for a review, see Downie et $a l^{1}$ and Egozcue et $a l^{2}$ ). These reports demonstrated the efficiency of the in situ labelling procedure on male gametes, but also pointed out the limitations of FISH on this biological material, which are essentially linked to the size of the probes and the reliability of the associated sperm decondensation treatments. ${ }^{2,3}$

The primed in situ (PRINS) reaction has offered an alternative approach for the direct chromosome analysis of human spermatozoa. Several studies have demonstrated that PRINS labelling was just as efficient as FISH on human sperm and exhibited higher specificity. ${ }^{4-6}$ The great

*Correspondence: Dr. F Pellestor, CNRS-UPR 1142, IGH, 141, rue de la Cardonille, F-34396 Montpellier cedex 5, France. Tel: 33 (0)4 996199 12; Fax: 33 (0)4 996199 01; E-mail: Franck.Pellestor@igh.cnrs.fr

Received 27 September 2002; revised 12 December 2002; accepted 12 December 2002 potential of this method based on the PCR principles, lies in the small size and the high specificity of the selected primers.

Peptide nucleic acid (PNA) probes provide a new and interesting alternative to FISH and PRINS. PNAs are synthetic mimics of DNA in which the deoxyribose phosphate backbone supporting the nucleic acid bases is replaced by a noncharged peptide backbone conferring greater stability and affinity to PNA probes than DNA probes. The efficiency of PNA probes has been demonstrated in the studies of telomere and centromere repeat sequences $^{7,8}$ and more recently for the specific in situ identification of human chromosomes. ${ }^{9}$ All of these reports have demonstrated the superiority of PNA probes over conventional repeat DNA probes in both signal intensity and sequence discrimination. However, no PNA probes have been yet produced for the detection of unique DNA sequences, such as telomere- and locus-specific sequences, for which efficient FISH probes are available. Consequently, we conducted a study to test PNA probes on human sperm preparations. In the present work, we report on the elaboration of an efficient multicolor PNA protocol for sperm nuclei analysis and the results of the first estimate of sperm disomy using this new type of probe. 


\section{Materials and methods PNA probes}

PNA probes specific for chromosomes $\mathrm{X}, \mathrm{Y}$ and 1 were kindly provided by Applied Biosystems (formerly Boston Probes Inc.). Each probe consists of a mixture of several short synthetic sequences (18-22 base units) specific for the centromeric tandem repeat sequence of the targeted chromosomes. The probe specific for chromosome $\mathrm{X}$ was labelled with fluorescein. The chromosome Y-specific probe was labelled with rhodamine and the chromosome 1 -specific probe was labelled in blue with diethylaminocoumarin. The PNA probes were supplied ready to use in hybridization buffer.

\section{Slide preparation}

Metaphase chromosomes were freshly prepared from peripheral blood lymphocytes by standard cytogenetic methods, fixed in methanol: glacial acetic acid (3:1) and spread on cleaned slides. Slides were passed through an ethanol series (70, 90, and 100\%), 2 min each step, and airdried. Slides were stored at room temperature and aged for at least $4 \mathrm{~h}$ at room temperature before proceeding with the hybridization steps. They were denaturated by immersion in $70 \%$ formamide, $2 \times \mathrm{SSC}$ at $72^{\circ} \mathrm{C}$ for $3 \mathrm{~min}$ and then dehydrated in a series of ice-cold ethanol washes $(70,90$ and $100 \%$ ) before being allowed to air-dry.

Sperm samples were obtained from two healthy males with normal peripheral blood karyotypes. Each ejaculate was collected in a sterile container and kept at room temperature for $30 \mathrm{~min}$. After liquefaction, an aliquot of the specimen was used for semen analysis. The rest of the sample was washed twice in $1 x P B S$ by centrifugation (five min at $2000 \mathrm{rpm}$ ) and fixed for one hour in fresh fixative (3:1 methanol:glacial acetic acid) at $4{ }^{\circ} \mathrm{C}$. The sperm suspension was then dropped onto clean microscope slides and air-dried. Slides were aged for 1 day at room temperature. At this step, two pretreatments were tested and sperm preparation slides from each ejaculate were divided into two groups. For the first group of slides, the simultaneous decondensation-denaturation $\mathrm{NaOH}$ protocol was used by immerging sperm slides in a $0.5 \mathrm{M} \mathrm{NaOH}$ solution at room temperature for $4 \mathrm{~min}$, followed by an ethanol series (70, 90 and 100\%) and air-drying. In the second group, a decondensing treatment based on dethiothreithol (DTT) was applied, consisting of the immersion of slides in a $1 \mathrm{M}$ DTT solution for $30 \mathrm{~min}$ at $4^{\circ} \mathrm{C}$, followed by two washes in $2 \times$ SSC ( $2 \mathrm{~min}$ ), an ethanol series and airdrying. The slides were then denaturated in $70 \%$ formamide, $2 \times \mathrm{SSC}$ at $72^{\circ} \mathrm{C}$ for $3 \mathrm{~min}$, dehydrated in a series of ice-cold ethanol washes (70, 90 and 100\%) and air-dried.

\section{Hybridization and posthybridization washes}

Probe aliquots of $5 \mu \mathrm{l}$ were mixed and denaturated for $6 \mathrm{~min}$ in a water bath preheated to $72^{\circ} \mathrm{C}$. The probe solution was applied to the preparation slide, covered with a $22 \times 22 \mathrm{~mm}$ coverslip and put in a humidified hybridization chamber 5-60 min at room temperature.

At the end of the hybridization, the coverslips were removed by washing of slides in $1 \times$ PBS, $0.1 \%$ Tween-20 for $2 \mathrm{~min}$. The slides were then transferred to a $58^{\circ} \mathrm{C}$ prewarmed $1 \times$ PBS, $0.1 \%$ Tween-20 for $20 \mathrm{~min}$ and rinsed in $2 \times$ SSC, $0.1 \%$ Tween-20 for $1 \mathrm{~min}$. The excess fluid was drained from the slides, which were then mounted in Vectashield antifade solution (Vector laboratories, Burlingame, CA, USA) containing a mixture of propidium iodide $(0.01 \mu \mathrm{g} / \mathrm{ml})$ and DAPI $(0.3 \mu \mathrm{g} / \mathrm{ml})$.

Disomy estimate: control, microscopic analysis, scoring criteria and statistical analysis

FISH and PRINS procedures were used as controls. Thus, in parallel with the PNA hybridizations, both FISH and PRINS estimates of disomy rates for chromosomes $\mathrm{X}, \mathrm{Y}$ and 1 were performed on sperm preparations from the same two donors. FISH assays were performed with the Vysis satellite probes CEPX, CEPY and CEP1 (Vysis, Downers Grove, IL, USA) directly labelled with the Spectrum Aqua ${ }^{\mathrm{TM}}$, Spectrum Green $^{\mathrm{TM}}$ and Spectrum Orange ${ }^{\mathrm{TM}}$, respectively. The FISH procedure was performed after DTT pre-treatment of sperm slides, according to the protocol recommended by Vysis, with an overnight hybridization at $37^{\circ} \mathrm{C}$. The PRINS assays were performed using $\mathrm{NaOH}$ pretreatment of sperm slides and primers specific for chromosomes $\mathrm{X}, \mathrm{Y}$ and 1 , as previously described. ${ }^{5,10}$ Their sequences and optimal technical conditions have been reported elsewhere. ${ }^{11}$

Two independent observers analyzed the slides, using a Leitz DMRB epifluorescence microscope (Leica SA, RueilMalmaison, France) equipped with appropriate excitation and emission filters. Each observer scored a minimum of 5000 sperm nuclei per slide. The haploid sperm nuclei displayed spots of different colors corresponding to each labelled chromosome. Nuclei were considered disomic when they displayed two signals similar in size and intensity, separated by at least the diameter of one fluorescent spot. ${ }^{12}$

The $\chi^{2}$ test was used to compare the disomy rates for the three chromosomes tested in each sperm sample, to compare this data between the two subjects and to test the variability of disomy values obtained with each of the three types of procedures (PNA, PRINS and FISH). For each donor, the homogeneity of the disomy rates, according to the labelling techniques used, was also tested by the Student's $t$-test. A value of $P<0.05$ was considered to be significant.

\section{Results}

The specificity and the labelling intensity of the PNA probes were first tested on metaphases and interphase nuclei using single- and two- or three-color PNA hybridizations. Examples of labelling on peripheral blood 


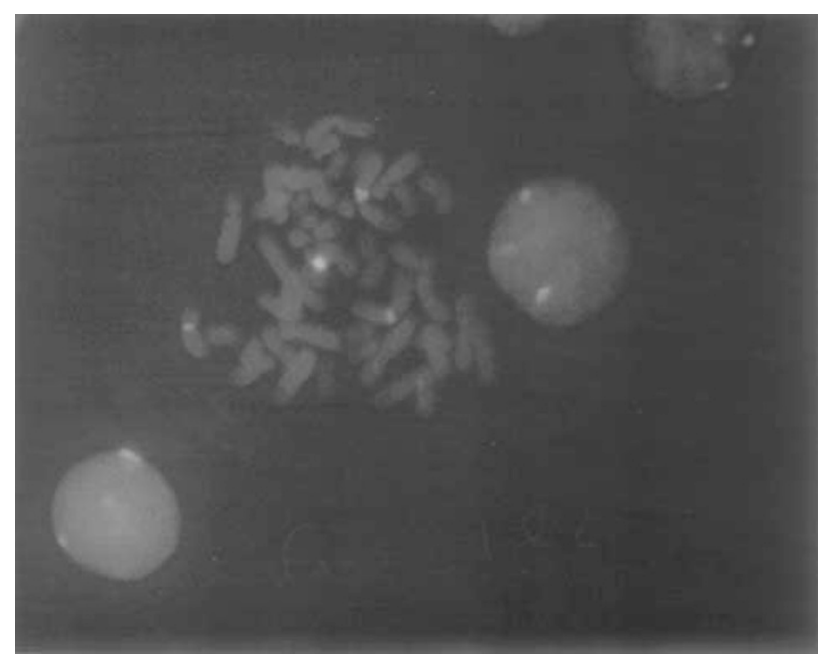

Figure 1 Three-color PNA labelling on human metaphase and interphase nuclei. Chromosome 1 is labelled in blue, chromosome $\mathrm{X}$ in red and chromosome $\mathrm{Y}$ in yellow.

lymphocytes are given in Figure 1. For each probe, resulting signals were specific and easily scorable. In double and triple hybridization experiments, the signals remained well defined without crosshybridization and significant background. The hybridization efficiency, estimated on 100 metaphases and nuclei, reached 100\% in single-color experiments and ranged from 97 to $100 \%$ in three-color PNA experiments. In order to assess the limits of PNA hybridization, several durations of hybridization time (from 5 to $60 \mathrm{~min}$ ) were tested. Satisfactory results, that is, well-distinguishable spots, were obtained in both metaphases and interphase nuclei following 20-60 min hybridization. The shortest hybridization did not allow enough time to obtain an efficient labelling of the targeted chromosomes.

On sperm preparation, the quality of the labelling was similar with the two decondensation pretreatments tested. As illustrated in Figure 2a, well-defined and bright signals were obtained on sperm nuclei without background, thus allowing an easy and reliable scoring of the targeted chromosomes. Several hybridization durations were tested on sperm preparations in order to define the minimal time of hybridization. With both DTT and $\mathrm{NaOH}$ pretretaments, the limit of in situ labelling was reached with a $25 \mathrm{~min}$ hybridization time.

According to these data, standard hybridization conditions on human sperm were fixed at $45 \mathrm{~min}$ with both $\mathrm{NaOH}$ and DTT pretreatments. The size of the fluorescent spot obtained was then similar to the size of the FISH signals, or the PRINS signals shown in Figure $2 \mathrm{~b}$.

Table 1 summarizes the results of the PNA estimates of disomy rates for chromosomes $\mathrm{X}, \mathrm{Y}$ and 1 in parallel with the estimates drawn from FISH and PRINS assays. In the PNA experiments, the ratio of $\mathrm{X}$ - and Y-bearing spermatozoa was $1: 1$ as expected. The frequencies were 0.14 and
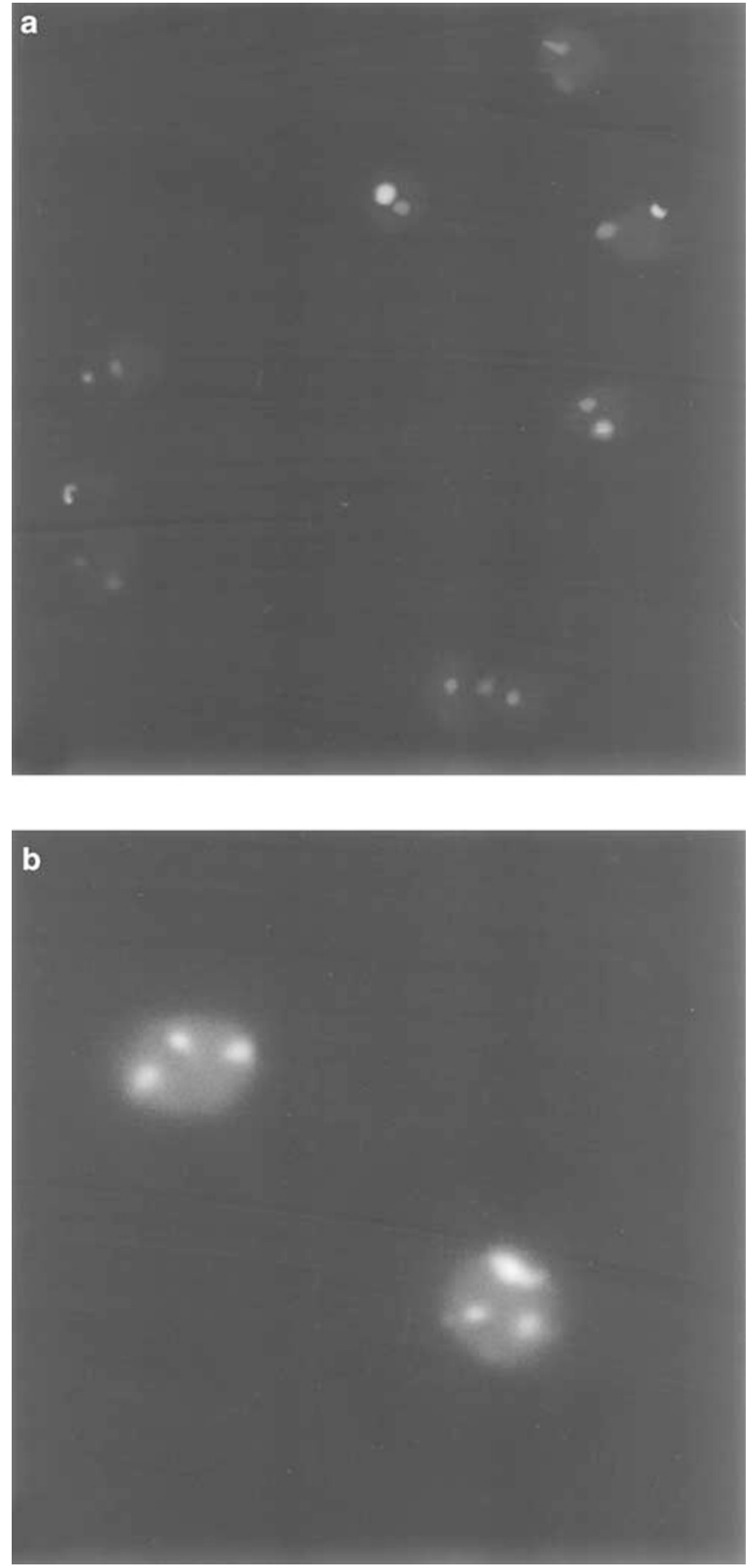

Figure 2 (a) Specific labelling of chromosome 1 (blue), $X$ (red) and $Y$ (yellow) on human sperm nuclei using PNA technique. (b) Detection of chromosome 1 (orange), chromosome X (red) and chromosome $Y$ (green) on human sperm nuclei using PRINS method.

$0.08 \%$ for XX disomy, 0.10 and $0.10 \%$ for YY disomy, 0.12 and $0.10 \%$ for $\mathrm{XY}$ disomy, and 0.13 and $0.18 \%$ for chromosome 1 disomy, in donors 1 and 2, respectively. The overall incidence of sex chromosome disomy (i.e. $\mathrm{XX}+\mathrm{YY}+\mathrm{XY}$ disomy rates) was $0.36 \%$ for donor 1 and 
Table 1 Results of sex ratio and disomy estimates for gonosomes and chromosome 1 using PNA, PRINS and FISH techniques on sperm preparations from two normal men

\begin{tabular}{|c|c|c|c|c|c|c|c|c|c|}
\hline \multirow[b]{2}{*}{ Methodology } & \multirow[b]{2}{*}{ Donor } & \multirow[b]{2}{*}{$\begin{array}{c}\text { No. of screened } \\
\text { nuclei }\end{array}$} & \multicolumn{2}{|c|}{ Sex ratio } & \multirow{2}{*}{$\begin{array}{c}\text { Overall sex } \\
\text { chromosome } \\
\text { disomy (\%) } \\
X X+Y Y+X Y\end{array}$} & \multirow[b]{2}{*}{$x x$} & \multicolumn{2}{|c|}{ Disomy } & \multirow[b]{2}{*}{ II } \\
\hline & & & $x$ & $Y$ & & & $Y Y$ & $X Y$ & \\
\hline PNA & 1 & 10008 & 4981 (49.77) & $4990(49.86)$ & 0.36 & $16(0.14)$ & $10(0.10)$ & $12(0.12)$ & $13(0.13)$ \\
\hline & 2 & 10036 & $5010(49.92)$ & 4998 (49.80) & 0.28 & $8(0.08)$ & $10(0.10)$ & $10(0.10)$ & $18(0.18)$ \\
\hline & 2 & 10013 & 5009 (50.03) & 5004 (49.97) & 0.32 & $9(0.09)$ & $10(0.10)$ & $13(0.13)$ & $21(0.21)$ \\
\hline
\end{tabular}

$0.28 \%$ for donor 2. Similar values were found in FISH and PRINS experiments (Table 1). These results are in agreement with the data previously reported in the literature.

No significant difference $(P>0.05)$ was observed between the disomy rates estimated by either PNA, FISH or PRINS for a same chromosome. The three approaches displayed no significant interindividual differences in gonosome disomy rates $(P>0.2)$ and chromosome 1 disomy rates $(P>0.3)$. For each patient, the comparative analysis of the PNA, FISH and PRINS data indicated no significant variation $(0.020<t<0.386 ; P>0.5)$. With the three procedures, a significant statistical difference was found between the overall sex chromosomes disomy rate and the chromosome 1 disomy rate in sperm from donor $1(P<0.01)$, but not in sperm from donor $2(P>0.05)$.

\section{Discussion}

The PNA probes present multiple advantages for the in situ detection of nucleic acid sequences. Several studies have already reported their successful use on metaphases and interphase nuclei for chromosomal identification. ${ }^{13,9}$ However, the adaptation of this new class of probes to human spermatozoa constituted a new step in the development of PNA methodology and a very interesting challenge because of the particularities of human sperm nucleus in terms of genomic compaction and accessibility of DNA sequences. On human sperm, two chromosomal labelling techniques have been successfully adapted, that is, the FISH and the PRINS methods. ${ }^{4,14}$ They constitute two well-distinct approaches for in situ chromosomal identification, and each presents advantages and disadvantages. Owing to its relative simplicity and the commercial availability of numerous probes, FISH has become the standard technique for sperm analysis, despite the lack of specificity of some alphoid probes. ${ }^{2}$ The PRINS reaction requires a thermocycler and sequential reactions, but provides a more specific and faster labelling procedure for the repeat DNA sequences., ${ }^{3,16}$ When applied to human sperm preparation, both techniques need to be associated with a sperm nucleus decondensing treatment in order to made the targeted DNA region accessible. The quality of the fluorescent signals, and consequently the reliability of the sperm analysis results are directly related to the efficiency of the in situ decondensation. ${ }^{17,18}$ Pretreatment to decondense sperm chromatin is also indispensable with PNA probes. No PNA labelling of sperm nuclei is obtained without decondensation pretreatment. We have tested the two decondensation procedures that give efficient and reproducible results with FISH and PRINS reactions, that is, the DTT and the $\mathrm{NaOH}$ treatments. The two procedures yielded satisfactory results with PNA and gave similar kinetics for the labelling reaction. The hybridization timing of PNA probes appeared to be considerably shortened in comparison with FISH reaction on sperm, which requires an overnight hybridization in order to be efficiently completed. The fast hybridization kinetics of PNA is more similar to the kinetics of PRINS reaction on which sperm chromosomal labelling can be performed in less than 20 min. ${ }^{10}$ The similarity between PNA and PRINS might be essentially because of the small size of both PNA oligoprobes (18-20 bp) and PRINS primers, which do not exceed 30 bases in length. Reduced labelling timing (around $3 \mathrm{~h}$ ) has also been reported in some sperm FISH studies, but using short satellite oligonucleotide probes. ${ }^{19,20}$ This data points out the importance of the probe size, and the use of a decondensation procedure in sperm labelling assays. Similar performance of PNA and PRINS methods has already been reported for the in situ detection and sizing of telomeric repeat sequences. ${ }^{21}$ Both techniques presented comparable features in terms of specificity, staining intensity and efficiency, but PRINS always displayed faster turnaround reaction time. This could reflect the fact that PRINS is an 'active' reaction involving an ultrafast biochemical reaction of primer extension catalyzed by a Taq polymerase. In the case of PNA reaction, the rapidity of the labelling is because of the neutral backbone of the PNA molecules, which allows for PNA/DNA binding to occur more rapidly and more tightly 
than DNA/DNA binding. Moreover, PNA probes can bind to DNA under low ionic strength conditions that disfavor reannealing of complimentary genomic strands. This advantage is particularly important for in situ hybridization experiments that target repetitive sequences, because both the length and the repetitive nature of the target sequences will affectively favor renaturation over hybridization with labelled probes. The high affinity of PNA probes to DNA constitutes an important feature for chromosomal analysis. Previous studies have demonstrated that PNA probes could discriminate between two centromeric DNA repeats that differ by only a single base pair. ${ }^{8,13}$ Identical results were obtained with PRINS primers ${ }^{22}$ and oligonucleotide probes, ${ }^{19}$ whereas standard FISH probes are unable to discriminate sequences with a single base resolution. The study of chromosomal polymorphism could benefit from the discriminating power of PNA. On sperm, this could be useful for the in situ distinction of autosomal nondisjunction occurring at meiosis I and meiosis II, when satellite polymorphisms exist between two homologous chromosomes. ${ }^{20}$

The PNA probes constitute new and valuable tools for in situ chromosomal investigation. They can advantageously be used to complement FISH and PRINS techniques and one can predict that the PNA technology is going to make significant progress during the next years. The successful testing of PNA probes on human sperm has proven that these reagents could be used on difficult biological material. Given their main features, that is, stability, specificity, rapidity, the PNA probes also have a great potential for clinical applications, in particular when chromosomal identification must be performed on limited amounts of material and in a limited time period such as in preimplantation chromosomal diagnosis.

\section{Acknowledgements}

This study was supported by the Association Française Contre les Myopathies (AFM) and an European grant COPERNICUS 2 (Contract ICA2-CT-2000-10012, proposal ICA2-1999-20007).

\section{References}

1 Downie SE, Flaherty SP, Matthews CD: Detection of chromosomes and estimation of aneuploidy in human spermatozoa using fluorescence in situ hybridization. Mol Hum Reprod 1997; 3: 58555898.

2 Egozcue J, Blanco J, Vidal F: Chromosome studies in human sperm nuclei using fluorescence in-situ hybridization (FISH). Hum Reprod Update 1997; 3: 441-452.

3 Guttenbach M, Engel W, Schmid M: Analysis of structural and numerical chromosome abnormalities in sperm of normal men and carriers of constitutional chromosome aberrations. A review. Hum Genet 1997; 100: 1-21.
4 Pellestor F, Girardet A, Lefort G, Andreo B, Charlieu JP: PRINS as a method for rapid chromosomal labeling on human spermatozoa. Mol Reprod Dev 1995; 40: 333-337.

5 Pellestor F, Girardet A, Coignet L, Andreo B, Charlieu JP: Assessment of aneuploidy for chromosomes 8, 9, 13, 16, and 21 in human sperm by using primed in situ labeling technique. Am J Hum Genet 1996; 58: 797-802.

6 Musio ASI: Detection of chromosome X in human sperm nuclei by direct and indirect primed in situ labeling (PRINS). Biochemica 1998; 2: 29-30.

7 Lansdorp PM, Verwoerd NP, van de Rijke FM et al: Heterogeneity in telomere length of human chromosomes. Hum Mol Genet 1996; 5: 685-691.

8 Chen C, Hong YK, Ontiveros SD, Egholm M, Strauss WM: Single base discrimination of CENP-B repeats on mouse and human chromosomes with PNA-FISH. Mamm Genome 1999; 10: 13-18.

9 Taneja KL, Chavez EA, Coull J, Lansdorp PM: Multicolor fluorescence in situ hybridization with peptide nucleic acid probes for enumeration of specific chromosomes in human cells. Genes Chromosomes Cancer 2001; 30: 57-63.

10 Pellestor F, Imbert I, Andreo B: Rapid chromosome detection by PRINS in human sperm. Am J Med Genet 2002; 107: 109-114.

11 Coullin P, Andreo B, Charlieu JP, Candelier JJ, Pellestor F: Primed in situ (PRINS) labelling with Alu and satellite primers for rapid characterization of human chromosomes in hybrid cell lines. Chromosome Res 1997; 5: 307-312.

12 Martin RH, Rademaker A: Reliability of aneuploidy estimates in human sperm: results of fluorescence in situ hybridization studies using two different scoring criteria. Mol Reprod Dev 1995; 42: 89-93.

13 Chen $\mathrm{C}$, Wu B, Wei T, Egholm M, Strauss WM: Unique chromosome identification and sequence-specific structural analysis with short PNA oligomers. Mamm Genome 2000; 11: 384-391.

14 Guttenbach M, Schmid M: Determination of Y chromosome aneuploidy in human sperm nuclei by nonradioactive in situ hybridization. Am J Hum Genet 1990; 46: 553-538.

15 Williams BJ, Ballenger CA, Malter HE et al: Non-disjunction in human sperm: results of fluorescence in situ hybridization studies using two and three probes. Hum Mol Genet 1993; 2: 1929-36.

16 Werner M, Wilkens L, Nasarek A, Tchinda J, Komminoth P: Detection of karyotype changes in interphase cells: oligonucleotide- primed in situ labelling versus fluorescence in situ hybridization. Virchows Arch 1997; 430: 381-387.

17 Wyrobek AJ: Methods and concepts in detecting abnormal reproductive outcomes of paternal origin. Reprod Toxicol 1993; 7 (Suppl 1): 3-16.

18 Martini E, Speel EJ, Geraedts JP, Ramaekers FC, Hopman AH Application of different in-situ hybridization detection methods for human sperm analysis. Hum Reprod 1995; 10: 855-861.

19 O'Keefe CL, Warburton PE, Matera AG: Oligonucleotide probes for alpha satellite DNA variants can distinguish homologous chromosomes by FISH. Hum Mol Genet 1996; 5: 1793-1799.

20 O'Keefe CL, Griffin DK, Bean CJ, Matera AG, Hassold TJ: Alphoid variant-specific FISH probes can distinguish autosomal meiosis I from meiosis II non-disjunction in human sperm. Hum Genet 1997; 101: 61-66.

21 Serakinci N, Koch J: Detection and sizing of telomeric repeat DNA in situ. Nat Biotechnol 1999; 17: 200-201.

22 Pellestor F, Girardet A, Lefort G, Andreo B, Charlieu JP: Rapid in situ detection of chromosome 21 by PRINS technique. Am J Med Genet 1995; 56: 393-397. 